\title{
Analysis of Electromagnetic Compatibility and Its Testing Technology Characteristics
}

\author{
Zhang Guoying, Ma Xingang, Zang Yuwei, Xie Lianke, Zhang Yong
}

State Grid Shandong Electric Power Research Institute, Jinan, Shandong, 250002

Keywords: electromagnetic compatibility, testing technology, test method, test site

\begin{abstract}
The analysis is carried out in this paper around electromagnetic compatibility and its testing technology characteristics to conduct in-depth research and exploration in electromagnetic compatibility, electromagnetic compatibility test equipment, electromagnetic compatibility test site and other fields, based on the development status of the current electromagnetic compatibility and its testing technology, aiming to better promote development and advancement of electromagnetic compatibility and its testing technology.

With the rapid development of social economy, the national life quality rises continuously and the use frequency and quantity of electronic and electrical devices are increasingly growing, which leads to a faster improvement of artificial space electromagnetic capability and heavy damage to the electromagnetic environment. Under the condition of such complicated electromagnetic environment, how to control the electromagnetic interference and promote scientific operation of the equipment is a key problem that needs to be solved at present. The fact that people pay more attention to electromagnetic environment and its influence also promotes rapid development and advancement of electromagnetic compatibility and its testing technology.
\end{abstract}

\section{Electromagnetic compatibility}

\subsection{Ideas}

Electromagnetic compatibility, also known as EMC, is widely used in LCD TV design, and also a key problem to be solved during LCD TV design. If there is a corresponding problem for the overall design of electromagnetic compatibility, corresponding amount of water ripples and blinking will be on the screen in the actual use of the LCD TV, and even images cannot be broadcast in serious cases. Electromagnetic compatibility design refers to the design made based on the actual requirements to reduce electromagnetic interference in electronic equipment, so that it fully meets the requirements of the computer compatibility standards. That is, the electromagnetic quantity produced by electronic equipment and related systems in the actual operation is controlled within the set standards and avoids any disruption to things and people in the neighborhood with perfect performance of anti-interference.

\subsection{Design criteria}

In order to better improve the electromagnetic compatibility of electronic equipment, designers should have a better understanding of the importance of electromagnetic compatibility based on the actual demand. In general, the design approach is regarded to fall into three points, which should be analyzed and studied, respectively: electromagnetic interference sources, electromagnetic interference routes and equipment vulnerable to electromagnetic interference, before scientific design in strict accordance with the previous design standards. First, designers should analyze and study the electromagnetic interference produced by the electrical equipment during the operation to acquire its related properties, adjust the parameters of the electromagnetic interference source, and keep it within the standard range established by each region. Second, designers should know and acquire the electrical interference routes, discover the interference and make mark in the first place, determine the actual interference route based on the relevant influence degree, reduce 
electromagnetic coupling and reduce electromagnetic interference in the route. Third, designers should makes an objective analysis of the electronic equipment which is more likely to be affected by electromagnetism, and take scientific measures to protect the related equipment to reduce the influence of electromagnetic interference on it.

\section{Devices for electromagnetic compatibility test}

In general, the spectrum analyzer based electromagnetic compatibility inspection system has the advantages of simplicity and precision and can provide various electromagnetic compatibility parameters quickly, so it is widely used. In addition, the organic integration of the spectrum analyzer and the brand new electromagnetic compatibility scanner really promotes the realization of electromagnetic radiation visualization. It can realize great $3 \mathrm{D}$ examination to the electronic equipment as well as a power supply unit in the system, PC board, cables and the like, truly displaying electromagnetic radiation of the equipment in a scientific manner.

The operation function of the interference receiving equipment with a mean and quasi-peak detector can better meet the requirements of the relevant rules and regulations. Normally, during the standard frequency range detection, two interference receiving equipment units with different frequency are required, with bands at $10 \mathrm{kHz}$ to $30 \mathrm{MHz}$ and $30 \mathrm{MHz}$ to $1000 \mathrm{MHz}$ respectively.

Besides, the artificial power network is also widely used in the electromagnetic compatibility test. During the test of the conduction interference current and voltage at the power supply end, operators need to use the artificial power network with electrical resistance at $50 \Omega / 50 \mu \mathrm{H}$, and its characteristics should meet the relevant standards fully. At this point, the artificial power network when connected and applied has two main roles: first, it provides great standard linear resistance for the equipment to be tested whose power supply is at the high frequency wave period, and there is no effect on the impedance of the input power of the device to be tested when it is connected to other equipment of the same power supply with corresponding changes. Second, the use of artificial power network can also better remove and filter the EMI coming from the power grid and provides AC mains supply for the switching power supply, with no effect on the conduction interference detection data existing in the equipment to be tested.

For different testing frequencies, operators can use the corresponding test antenna. When the frequency band is between $10 \mathrm{kHz}$ and $30 \mathrm{MHz}$, the annular antenna with shielding function can be used; when the frequency band is $30 \mathrm{MHz}$ to $1000 \mathrm{MHz}$, the dipole antenna can be used.

\section{Electromagnetic compatibility test site}

In the electromagnetic compatibility test by relevant operators, the site conditions usually have a direct impact on the test data. The data of the same equipment tested will be different when the test is carried out at different sites. The main reason is that there is a big gap between the reflected waves at different test sites; as a result, the field strengths on top of one another are greatly different.

\subsection{Open test site}

The main feature of the open test site is that the vicinity is extremely open, there is no reflective object, the ground is flat and the metal conductor is grounded scientifically. The test site should be oval shaped on the whole with length greater than two times the distance between focuses of the ellipse and width greater than one time such distance. Its size should be determined based on the wavelength in the lower limit test frequency. During the electromagnetic compatibility radiation test, the equipment tested and the receiving antenna should be installed scientifically at the focuses of the ellipse. The open test site is mainly suitable for electromagnetic compatibility test at a frequency of $30 \mathrm{MHz}$ to $1000 \mathrm{MHz}$. During the test of electromagnetic radiation interference of the tested equipment, the open test site can be used for scientific examination and research of large equipment. And the open test site can also be used to detect the interference rejection. However, it cannot be given large field strength in the actual test to avoid producing corresponding electromagnetic 
interference to the outside world.

\subsection{Shielded chamber}

The shielded chamber is a large-scale hexahedron mainly constructed with metal plates and metal meshes. Because the metal material has a strong shielding function, the test carries out in the shielded chamber will not be affected by the external electromagnetic environment influence and the interference. Besides, the sensitivity to the large field strong electromagnetic radiation can also be tested in the shielded chamber with no electromagnetic interference to the external environment. From the macroscopic point of view, the shielded chamber is mainly a large-scale rectangular waveguide resonator cavity and it will produce resonance at the corresponding frequency, at this point, the relevant functions of the shielded chamber will be seriously affected, and even there will be errors in the test result. The main method to reduce the resonance of the shielded chamber is to control the quality factors scientifically.

\subsection{EM chamber}

The so-called EM chamber is mainly constructed by installing EM wave absorption materials completely in the shielded chamber to absorb the electromagnetic field reflected to the shielded chamber walls and remove reflection of the walls. The EM chamber is also known as anechoic chamber. Compared with the shielded chamber, it costs higher in construction, but it can simulate the environment with no electromagnetic pollution or reflection. During the electromagnetic compatibility test, the semi-EM chamber is an ideal test site. The main difference is that the shielded chamber floor is not equipped with the corresponding wave-absorbing material.

\subsection{G Hertz transverse electromagnetic wave chamber}

G Hertz transverse electromagnetic wave (GTEM) chamber was put forward in the early 70's by researchers in the United States based on the idea of a rectangular coaxial axis. Its essence is to expand and extend the coaxial transmission line by a scientific means, which can transfer the transverse wave more evenly inside and simulate the plane wave in free space. From the macroscopic point of view, it is a movable shielded chamber, which can be conveniently operated. During the electromagnetic compatibility test in the GTEM chamber, the chamber should fully meet the $1 / 3$ principle. If it fails to meet the demand, the uniformity of the test site will be seriously affected and its resistance is also lack of matching, ultimately leading to the decreased accuracy of the test data. And the GTEM chamber is also an ideal site for the detection of electromagnetic compatibility radiation sensitivity of the electronic equipment. The small working space of the GTEM chamber and reflection in the high frequency transition period lead to the high frequency constraint in the GTEM chamber, which usually should be within $500 \mathrm{MHz}$.

\section{Electromagnetic compatibility test method}

\subsection{Direct test method}

The so-called direct test method is to carry on the electromagnetic compatibility test at the test site that the corresponding electromagnetic compatibility standard requires. The test result is more accurate, but it costs higher. During the test at the site, the equipment to be tested needs to be located in the same state as in the radiation measurement, and the equipment to be tested should also be located with a minimum extension of $0.5 \mathrm{~m}$ from its boundary value or on a metal connecting floor sized $2 \mathrm{~m} \times 2 \mathrm{~m}$. During the electromagnetic compatibility test in the shielded chamber, operators can use the ground shielding layer and any wall shield as the grounding plate facility. Besides, there are strict rules and standards during the test arrangement. For example, non-landing equipment to be tested should be mounted on the platform and the insulation support at $0.4 \mathrm{~m}$ above the ground; the landing equipment to be tested should be placed directly on the grounding plate with strong insulation measures for its mutual contact position or the same as in the use. 


\subsection{To-be-tested method}

During the use of the direct test method, there is a high demand for the lab configuration, and it is difficult for the enterprise to meet the cost requirement if the test site is needed. However, the to-be-tested method can lower the corresponding costs to the maximum extent while ensuring the comparable test results, so it is well received by lots of enterprises. The researchers have suggested that the absorption clamp method specified in the relevant testing standards for the use of larger electronic devices should be comprehensively and scientifically tested on the external radiated power of the equipment. The absorption clamp method can be used to test the conduction interference equipment at a frequency of $5 \mathrm{MHz}$ to $300 \mathrm{MHz}$. This method requires a small size of the equipment to be tested, and the energy that produces radiation is usually generated by using the power cord of the equipment to be tested. This method is mainly characterized by advantages such as simple and flexible operation and less demand for the environment. Based on this method, corresponding tests can be carried out in the shielded chamber simply. Besides, the alternative test method is also highly credible in the craft and its process is extremely simple with a low configuration cost.

\subsection{Automatic test method}

In this paper, an automatic test method is used as an example for analysis. The repeater is mainly in the linear state during the operation and its gain is adjusted to the highest. The power output of the noise coefficient detection equipment is connected to the noise generation equipment for adjustment and driving. The output end of the noise generation equipment is connected with the input end of the noise coefficient detection equipment to scientifically adjust and correct the noise coefficient detection equipment. When the tested repeater is connected to the noise generation equipment and the noise coefficient detection equipment, operators can display the noise parameters of the tested repeater directly on the noise coefficient detection equipment. When conditions permit, the use of the automatic test method should be allowed.

\section{Conclusion}

In conclusion, during the use of electromagnetic compatibility and its testing technology, relevant operators should pay great attention to the electromagnetic compatibility test equipment, electromagnetic compatibility test methods and electromagnetic compatibility test sites. Only in this way, can the accuracy of the electromagnetic compatibility test results be bettered and the actual electromagnetic environment be improved to a certain extent.

\section{References}

[1] Chen Yong, Zhang Cancan, Li Fuqiu, Huang Zhen, Bian Mengyun, Chen Qi, Xu Lingfang, Li Zhang, Research on LED Illumination EMC and Test Technolog [J]. Information \& Communication, 2016 (08).

[2] Jia Qiucheng. Research on EMC Test Technology of Mobile Phone [D]. Suzhou University, 2015.

[3] Chen Lu. EMC Field Automatic Test Technology [D]. Xidian University, 2014.

[4] Li Zhiyuan. Seminar on System-level EMC Test Technology Held by R\&S [J]. Telecommunications Network Technology, 2010 (08).

[5] Wang Yuayuan, Xu Qiong, Tong Jun. Overview of EMC Test Technology for Integrated Circuits [J]. Silicon Valley, 2008 (19).

[6] Chen Weifeng, Liu Weilian, Zhou Xiang. EMC and Its Testing Technology [J]. Electronic Measurement Technology, 2008 (01).

[7] Zhu Zhiyu, Zhang Bing, Liu Witing. Research on Ship EMC Testing Technology Based on Multiresolution Wavelet Analysis [J]. Navigation of China, 2005 (03). 